\title{
IDO inhibitor synergized with radiotherapy to delay tumor growth by reversing $\mathbf{T}$ cell exhaustion
}

\author{
MENG LIU ${ }^{1,2^{*}}$, ZIYANG LI ${ }^{1,2^{*}}$, WEIRONG YAO ${ }^{3}$, XIAOPING ZENG ${ }^{1}$, LINGYUN WANG $^{1}$, \\ JIAO CHENG ${ }^{1}$, BINGYU MA ${ }^{1,2}$, RUIQIAN ZHANG ${ }^{1,2}$, WEIPING MIN ${ }^{1,4}$ and HONGMEI WANG ${ }^{1}$ \\ ${ }^{1}$ Departments of Pathophysiology and Immunology, School of Basic Medical Sciences, Nanchang University; \\ ${ }^{2}$ Academy of Queen Mary, Nanchang University; ${ }^{3}$ Department of Oncology, Jiangxi Provincial People's Hospital; \\ ${ }^{4}$ Jiangxi Provincial Key Laboratory of Immunotherapy, Jiangxi Academy of Medical Sciences, \\ Nanchang, Jiangxi 330006, P.R. China
}

Received April 19, 2019; Accepted September 26, 2019

DOI: $10.3892 / \mathrm{mmr} .2019 .10816$

\begin{abstract}
Previous studies suggest that radiotherapy (RT) can induce immune activation, which not only reduces the progression of tumors, but also causes the release of tumor antigens. The combination of RT and immune checkpoint blockade, such as the inhibition of programmed cell death 1 (PD-1) and programmed cell death ligand 1 (PD-L1), has been demonstrated to yield impressive response rates. However, a substantial proportion of patients develop resistance such therapies. Previous studies have shown that indoleamine 2,3-dioxygenase (IDO) causes T cell exhaustion and increased formation of regulatory $\mathrm{T}$ cells (Tregs), upregulating the expression of inhibitory receptors and ligands. Therefore, the application of IDO inhibitors combined with RT may have a synergistic effect by relieving immunosuppression. Lewis lung cancer cell-bearing mice were treated with the IDO inhibitor 1-methyl-tryptophan (1MT) and/or 10 Gy RT. Tumor size was measured every day, flow cytometry was performed to measure the expression of dendritic cell (DC) maturation markers, inhibitory receptors, ligands, cytotoxic $\mathrm{T}$ cells and Treg phenotypic markers. Reverse transcription-quantitative PCR was used to evaluate the mRNA expression levels of IDO, PD-L1, PD-1, T cell immunoglobulin domain and mucin domain 3 (TIM-3), B- and T-lymphocyte attenuator (BTLA) and galectin-9. Compared with the control group, treatment with 1MT or RT reduced tumor growth, however, the combination therapy was more effective than either treatment alone.
\end{abstract}

Correspondence to: Dr Hongmei Wang, Departments of Pathophysiology and Immunology, School of Basic Medical Sciences, Nanchang University, 461 Bayi Avenue, Nanchang, Jiangxi 330006, P.R. China

E-mail: wanghongmay@hotmail.com

*Contributed equally

Key words: indoleamine 2,3-dioxygenase, 1-methyl-tryptophan, radiotherapy, immunotherapy, Lewis lung cancer
Flow cytometry showed the upregulation of CD80, CD86 and the major histocompatibility complex II in spleen DCs and the concurrent downregulation of CD4, CD25 and forkhead box protein $\mathrm{P} 3$ in lymphocytes in the treatment groups. Compared with the control group, inhibitory receptors and ligands that affect DCs and T cells were observed, expression levels of PD-L1, PD-1, TIM-3, BTLA and galectin-9 are decreased in treatment group compared with control. IDO inhibition synergized with RT to downregulate Tregs, inhibitory receptors and ligands to prevent $T$ cell exhaustion. By activating DCs and $\mathrm{T}$ cells, this combination therapy may strongly enhance antitumor immunity and inhibit tumor progression.

\section{Introduction}

Radiotherapy (RT) is widely used to treat cancer because of its ability to control tumor growth; RT can destroy cancerous tissue and cause tumor cell necrosis (1). RT induces changes in the tumor microenvironment and promotes the expression, and release of, tumor-related antigens, thereby activating the antitumor immune response (2). RT also induces tumor cells to produce cytokines, such as tumor necrosis factor (TNF)- $\alpha$, which attract effector $T$ cells to the target region, increasing the expression of damage-associated molecular patterns on the surface of tumor cell, which induces 'immunogenic cell death' in tumor cells $(3,4)$. Previous studies have reported that RT can upregulate the expression of CD70 on dendritic cells (DCs) and induce $\mathrm{T}$ cells to produce interferon (IFN)- $\gamma(5,6) . \mathrm{CD} 8^{+} \mathrm{T}$ cells are activated after RT combined with immunotherapy (7). Together, RT and immunotherapy increase the antigen presenting ability of DCs and increase the number of antigens on the surface of tumor cells, inducing T cell-mediated clearance $(8,9)$.

However, the upregulation of regulatory T cells (Tregs), and some inhibitory receptors and ligands, including programmed cell death 1 (PD-1), programmed cell death ligand 1 (PD-L1), $\mathrm{T}$ cell immunoglobulin domain and mucin domain 3 (TIM-3), B- and T-lymphocyte attenuator (BTLA) and galectin-9, prevents RT from achieving the optimal therapeutic effect (10). These molecules are major biological markers of $\mathrm{T}$ cell exhaustion, which leads to reduced $\mathrm{T}$ cell proliferation and cytotoxicity, and increased apoptosis. The 
antitumor cytotoxic effect and the adaptive immunotherapy effect of T cells is reduced, which leads to the occurrence, development and metastasis of tumors (11). Therefore, RT combined with immune checkpoint blockade has attracted considerable attention and has already achieved some success. PD-1 blockade combined with RT is among the most effective immunotherapies for cancer $(12,13)$; however, a substantial proportion of patients gradually develop treatment resistance (14-16). Currently, the mechanism of drug resistance remains uncertain, reflecting the need for a new strategy (17).

One potential explanation for tumor immune escape and resistance involves the immaturity of DCs, and the exhaustion of T cells $(18,19)$. Indoleamine 2,3-dioxygenase (IDO) is an important regulatory factor in tumor-mediated immunosuppression that has a negative effect on DCs and T cells (20).

As the tryptophan catabolic enzyme involved in the initiation and rate-limiting steps, IDO is highly expressed in various types of cancer, as well as in immune cells, and plays an important role in immune cell suppression $(15,21)$. In humans and murine models, the expression of IDO has been found to be a prominent predictor of a poor prognosis (19), which may be related to its negative regulatory effect on DCs and T cells. IDO overexpression in DCs can affect their maturation, resulting in a decreased antigen-presenting ability and increased negative co-stimulatory molecule expression. These effects also allow IDO to indirectly influence local T cells (20). Additionally, IDO supplementation induces Tregs, which contribute to the immunosuppressive effects (22). IDO can mediate the depletion of the essential amino acid tryptophan, causing tryptophan starvation (11). In the middle of the G1 phase, T cells are extremely sensitive to tryptophan deficiency, which causes protein insufficiency and blockade in the G1 phase. This effect can increase the susceptibility of T cells to apoptosis (14). Together, the negative effects of IDO on DCs and $\mathrm{T}$ cells leads to immunosuppression in tumor cells.

Researchers have expressed interest in developing IDO inhibitors as new immunotherapies to treat cancer (23). Although 1-methyl-tryptophan (1MT) has not achieved the desired anticancer effect in recent clinical trials (23), as a general therapeutic approach, IDO inhibitors still have potential and research value. IDO inhibition combined with RT may provide a better therapeutic effect, and may partially solve the problem of tumor immunosuppression. The present study aimed to investigate whether an IDO inhibitor synergized with RT can further inhibit tumor growth by activating DCs and antagonizing $\mathrm{T}$ cell exhaustion.

\section{Materials and methods}

Cell line and animals. Lewis lung cancer (LLC) cells, a murine tumor cell line, were obtained from the American Type Culture Collection and maintained in RPMI 1640 medium (HyClone; GE Healthcare Life Sciences) with 10\% FBS (Gibco; Thermo Fisher Scientific, Inc.), $100 \mathrm{U} / \mathrm{ml}$ penicillin (Gibco; Thermo Fisher Scientific, Inc.) and $100 \mu \mathrm{g} / \mathrm{ml}$ streptomycin (Gibco; Thermo Fisher Scientific, Inc.) at $37^{\circ} \mathrm{C}$ in $5 \% \mathrm{CO}_{2}$. The media was changed every other day and the cells were digested with $0.25 \%$ trypsin for 2-3 min in the logarithmic growth phase from passages 1-4. Mycoplasma testing was completed for the cell line.
C57BL/6 female mice (age, 6-8 weeks old; weight, 20-25 g) were purchased from the animal breeding facility of Hunan Slack Jingda Experimental Animal Co., Ltd. (cat. no. 43004700049723). The mice were maintained under controlled conditions $\left(20-23^{\circ} \mathrm{C}, 40-70 \%\right.$ humidity and a $12-\mathrm{h}$ light/dark cycle). Mice ate $\sim 5 \mathrm{~g}$ chow per day and drank $\sim 7 \mathrm{ml}$ water. All animal experiments were performed in accordance with the Animal Research Reporting of in vivo Experiments and the National Institutes of Health guidelines for animal welfare, and the study was approved by the Laboratory Animal Ethics Committee of Nanchang University (permit no. NCDXSYDWFL-2015097). All animal experiments complied with the ARRIVE guidelines and the 2013 AVMA euthanasia guidelines (24).

Tumor models and treatment protocols. Tumors were generated on the backs of mice by subcutaneously injecting $1 \times 10^{5}$ LLC cells in PBS. Tumor size was measured using a caliper every day after the tumors appeared, and the tumor volume was calculated using the following formula: Tumor volume $=0.5 \mathrm{x}$ (tumor length) $\mathrm{x}$ (tumor width) ${ }^{2}$. The mice were monitored daily and euthanized using $\mathrm{CO}_{2}$ inhalation (the flow rate of $\mathrm{CO}_{2}$ was $20 \%$ of the chamber volume/minute). Mice were euthanized if any of the following conditions were observed: A body condition score of 1/5 (mouse is emaciated: Skeletal structure is extremely prominent; little or no flesh cover, or vertebrae distinctly segmented), a body condition score of $2 / 5$ (mouse is under conditioned: Segmentation of the vertebral column evident, or dorsal pelvic bones are readily palpable) and profound lethargy, a tumor affecting the gait, normal posture or ability to eat, urinate, or defecate independently of the size of the tumor or the determination by a University Laboratory Animal Resources veterinarian that the animal should be euthanized. Animal death was verified by pupil dilation and the absence of a heartbeat. The mice were divided into 4 groups: i) The control group (CON); ii) the RT group; iii) the 1MT group; and iv) the combined group (1MT + RT). On the first day after tumor inoculation, 1MT was diluted to $400 \mathrm{mg} / \mathrm{kg}$ in saline and was administered by gavage twice daily. On day 13 after inoculation of the tumor, RT was given locally on the mice in the RT group with a fractionation scheme of 10 Gy [the mice were anesthetized with chloral hydrate ( $4 \%$ solution, $400 \mathrm{mg} / \mathrm{kg}$ ) by intraperitoneal injection before receiving radiation].

The mice were sacrificed on day 28 after inoculation. Tumors were recovered and weighed, and tumors and spleens from each group were quickly prepared for flow cytometry. Tumor-bearing mouse spleens were disaggregated with the flat end of a syringe in $5 \mathrm{ml}$ of RPMI 1640 medium in a tissue culture dish. Dispersed cells were filtered through a $40-\mu \mathrm{m}$ Falcon cell strainer.

Flow cytometry analysis. Phenotypic analysis and characterization of DCs or T cells was performed using a FACSCanto II flow cytometer (BD Biosciences). Antibodies were purchased from eBioscience; Thermo Fisher Scientific, Inc. Individual spleens were isolated and pressed through a $40-\mu \mathrm{m}$ Falcon Cell Strainer. Dissociated cells were treated with Ammonium-Chloride-Potassium lysis buffer (Lonza Group 
Ltd.) to lyse red blood cells. The resulting cell suspension was then centrifuged for $5 \mathrm{~min}$ at $500 \mathrm{x} \mathrm{g}$ and $4^{\circ} \mathrm{C}$, before removal of the supernatant and re-suspension of the cells in PBS for further FACS analysis. DC and T cell subsets were analyzed with two- or three-color staining with various combinations of mouse antibodies. DCs were stained with FITC-CD11C (cat. no. 11-0041-85), phycoerythrin (PE)-CD80 (cat. no. 12-0081-82), PE-CD86 (cat. no. 12-0861-83) and PE-major histocompatibility complex (MHC) II (cat. no. 1895-09) monoclonal antibodies. For T cells, FITC-CD4 (cat.no.11-0041-82), allophycocyanin(APC)-CD25 (cat. no. 12-0251-83), PE-FOXP3 (cat. no. 563101), PE-CD8 (cat. no. 12-0081-82) and APC-IFN- $\gamma$ (cat. no. 17-7311-82) conjugated anti-mouse monoclonal antibodies were used for staining $\left(3 \times 10^{5}\right.$ cells in $150 \mu \mathrm{l}$ full medium with $0.2 \mu \mathrm{g}$ antibodies; all antibodies and isotype controls were diluted using this method). FOXP3 and IFN- $\gamma$ expression was assessed using intracellular staining with Fixation/Permeabilization Concentrate and Fixation/Permeabilization Diluent (eBioscience; Thermo Fisher Scientific, Inc.). Isotype controls (mouse IgG2a K isotype control-PE, cat. no. 43912-60-100; mouse IgG1 K isotype control-APC, cat. no. 44212-80-100; mouse IgG2a K isotype control FITC, cat. no. 43912-50-100) were used to discriminate positive cell staining from nonspecific background staining.). Results were analyzed using FlowJo version 10.5.2 (FlowJo LLC) software.

Reverse transcription-quantitative $(R T-q) P C R$. Total RNA was isolated using TRIzol ${ }^{\circledR}$ (Invitrogen; Thermo Fisher Scientific, Inc.) and complementary (c)DNA synthesis was performed using the Vazyme Reverse Transcription System (Vazyme). HiScript II qRTSuperMix II was added to RNA, which was reverse transcribed using the following temperature protocol: $10 \mathrm{~min}$ at $25^{\circ} \mathrm{C}, 30 \mathrm{~min}$ at $42^{\circ} \mathrm{C}$ and $5 \mathrm{~min}$ at $85^{\circ} \mathrm{C}$. The mRNAs of mouse IDO, PD-1, PD-L1, TIM-3, BTLA and galectin-9 were determined using RT-qPCR. Using the RT reaction as a template, SYBR-Green I dye (Vazyme) was used for qPCR. The reaction conditions were $10 \mathrm{~min}$ at $95^{\circ} \mathrm{C}$, followed by $15 \mathrm{sec}$ at $95^{\circ} \mathrm{C}$ and $1 \mathrm{~min}$ at $60^{\circ} \mathrm{C}$ for 40 cycles for IDO, or $1 \mathrm{~min}$ at $58^{\circ} \mathrm{C}$ for 40 cycles for $\mathrm{PD}-1$, or $1 \mathrm{~min}$ at $63^{\circ} \mathrm{C}$ for 40 cycles for PD-L1, or $1 \mathrm{~min}$ at $60^{\circ} \mathrm{C}$ for 40 cycles for TIM-3, BTLA and galectin-9. The primer sequences for RT-qPCR are listed in Table I. The relative mRNA expression levels of target genes were calculated using the $2^{-\Delta \Delta C a}$ method (25) compared with GAPDH expression.

Statistical analysis. Experiments were repeated three times. Data are expressed as the mean \pm SD. Comparisons among groups were performed using One-way ANOVA followed by Tukey's post hoc test. Data analysis was performed using SPSS 17.0 software (SPSS, Inc.). $\mathrm{P}<0.05$ was considered to indicate a statistically significant difference.

\section{Results}

IDO inhibition combined with RT downregulates IDO expression and suppresses tumor growth. To examine whether IDO inhibition affects tumor growth, mice were divided into four groups and subjected to various treatments. The longest diameter of a single subcutaneous tumor was $1.9 \mathrm{~cm}$ and no animal presented with multiple tumors. The growth curve (Fig. 1A) suggested that tumor progression was significantly slower in both the RT group and the 1MT group compared with the CON group. Furthermore, 1MT combined with RT had a larger therapeutic effect than either treatment alone. RT-qPCR showed that the tumors in the 1MT and the combined groups had lower IDO expression levels than those in the CON group (Fig. 1B), suggesting that differences in the therapeutic outcome may be due to differences in IDO expression. On the 28th day after tumor inoculation, the mice were euthanized using carbon dioxide inhalation and the tumor tissue was weighed. The tumor weights and data are shown in Fig. 1C and D. As shown in Fig. 1D, the average tumor weights were as follows: $2.165 \mathrm{~g}$ in the CON group, $0.834 \mathrm{~g}$ in the RT group, $0.324 \mathrm{~g}$ in the $1 \mathrm{MT}$ group and $0.107 \mathrm{~g}$ in the combined group.

The slower tumor progression in the 1MT-treated group compared with the CON group suggested that IDO is responsible, at least in part, for tumor immunosuppression and that using the 1MT inhibitor can reverse this effect. The combined group showed a significantly better therapeutic effect than the other groups, indicating that $1 \mathrm{MT}$ and RT can act synergistically. Some of the mechanisms driving this effect are discussed below.

IDO inhibition synergizes with RT to promote the maturation of DCs and downregulate the inhibitory receptor ligand PD-L1 in the tumor microenvironment. As shown in Fig. 1, compared to the control condition, combined therapy delays tumor progression. One potential explanation for tumor immune escape and resistance is that IDO may be involved in DC immaturity and T cell anergy, suggesting that the inhibition of IDO expression induced by 1MT may lead to the tumor suppressive effects shown in the Fig. 1. To investigate the influences of changes in IDO on DCs, maturity markers, including CD80, CD86 and MHC II, were examined. Based on flow cytometry analysis (Fig. 2A-C), the results indicated that these co-stimulatory factors were highly expressed in the combined treatment group, with $\mathrm{CD}^{2} 0^{+}$expression in $80.1 \%$

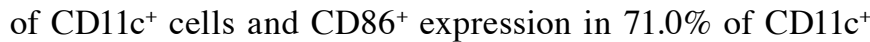
cells. Additionally, the DCs in the 1MT group and RT group also showed a high degree of maturity, indicating that RT and IDO inhibition increased the maturity of DCs, and that these therapies can synergize to produce greater effects.

Mature DCs must express high levels of MHC II and downregulate co-inhibitory molecules to initiate $\mathrm{T}$ cell activation, and provide a secondary stimulatory signal. Based on flow cytometry analysis (Fig. 2A and D), DCs in the RT, 1MT and combined groups showed high MHC II expression, with the mean values of $89.27 \pm 1.71,95.00 \pm 0.50$ and $96.63 \pm 0.54 \%$

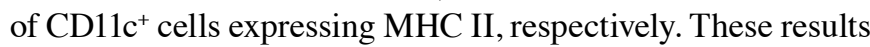
indicated that RT and IDO inhibition upregulate the expression of MHC II, which may increase the antigen-presenting ability of DCs. These treatments also showed increased therapeutic effects when combined compared with their use alone. RT-qPCR showed that PD-L1 in the tumor microenvironment, as an inhibitory receptor ligand, was significantly downregulated after RT and/or 1MT treatment compared with the corresponding level in the CON group (Fig. 2E), which reduces the inhibitory effect of PD-L1 on T cells. These results indicated that RT and IDO inhibition can lead to the progression of 
Table I. Sequences of primers used for reverse transcription-quantitative PCR.

\begin{tabular}{|c|c|c|}
\hline Gene & Primer & Product length \\
\hline \multirow[t]{2}{*}{ IDO } & F 5'-GGGCTTTGCTCTACCACATCCACT-3' & $234 \mathrm{bp}$ \\
\hline & R 5'-ACATCGTCATCCCCTCGGTTCC-3' & \\
\hline \multirow[t]{2}{*}{ BTLA } & F 5'-TGCAGGAGCCAGAAGAGAAAGTCA-3' & $306 \mathrm{bp}$ \\
\hline & R: 5'-CAATGTGGGGGTCAGGGATGG-3' & \\
\hline \multirow[t]{2}{*}{ GAL-9 } & F 5'-GTTGTCCGAAACAACTCAGAT-3' & $315 \mathrm{bp}$ \\
\hline & R 5'-ATATGATCCACACCGAGAAG-3' & \\
\hline \multirow[t]{2}{*}{ PD-1 } & F 5'-GGCCGCCTTCTGTAATGGTTTGA-3' & 279 bp \\
\hline & R 5'-AGGGGCTGGGATATCTTGTTGAGG-3' & \\
\hline \multirow[t]{2}{*}{ PD-L1 } & F 5'-GACCAGCTTTTGAAGGGAAATG-3' & $385 \mathrm{bp}$ \\
\hline & R 5'-CTGGTTGATTTTGCGGTATGG-3' & \\
\hline \multirow[t]{2}{*}{ TIM3 } & F 5'-AGTGGGAGTCTCTGCTGGGTTGA-3' & $279 \mathrm{bp}$ \\
\hline & R 5'-AGGATGGCTGCTGGCTGTTGA-3' & \\
\hline \multirow[t]{2}{*}{ GAPDH } & F 5'-TGATG ACATCA AGAAGGTGGTGAA-3' & $345 \mathrm{bp}$ \\
\hline & R 5'-TGGGATGGAA ATTGTGAGGGAGAT-3' & \\
\hline
\end{tabular}

IDO, indoleamine 2,3-dioxygenase; BTLA, B- and T-lymphocyte attenuator; GAL-9, galectin-9; PD-1, programmed cell death protein-1; PD-L1, programmed cell death ligand-1; TIM3, T cell immunoglobulin-3; F, forward; R, reverse.
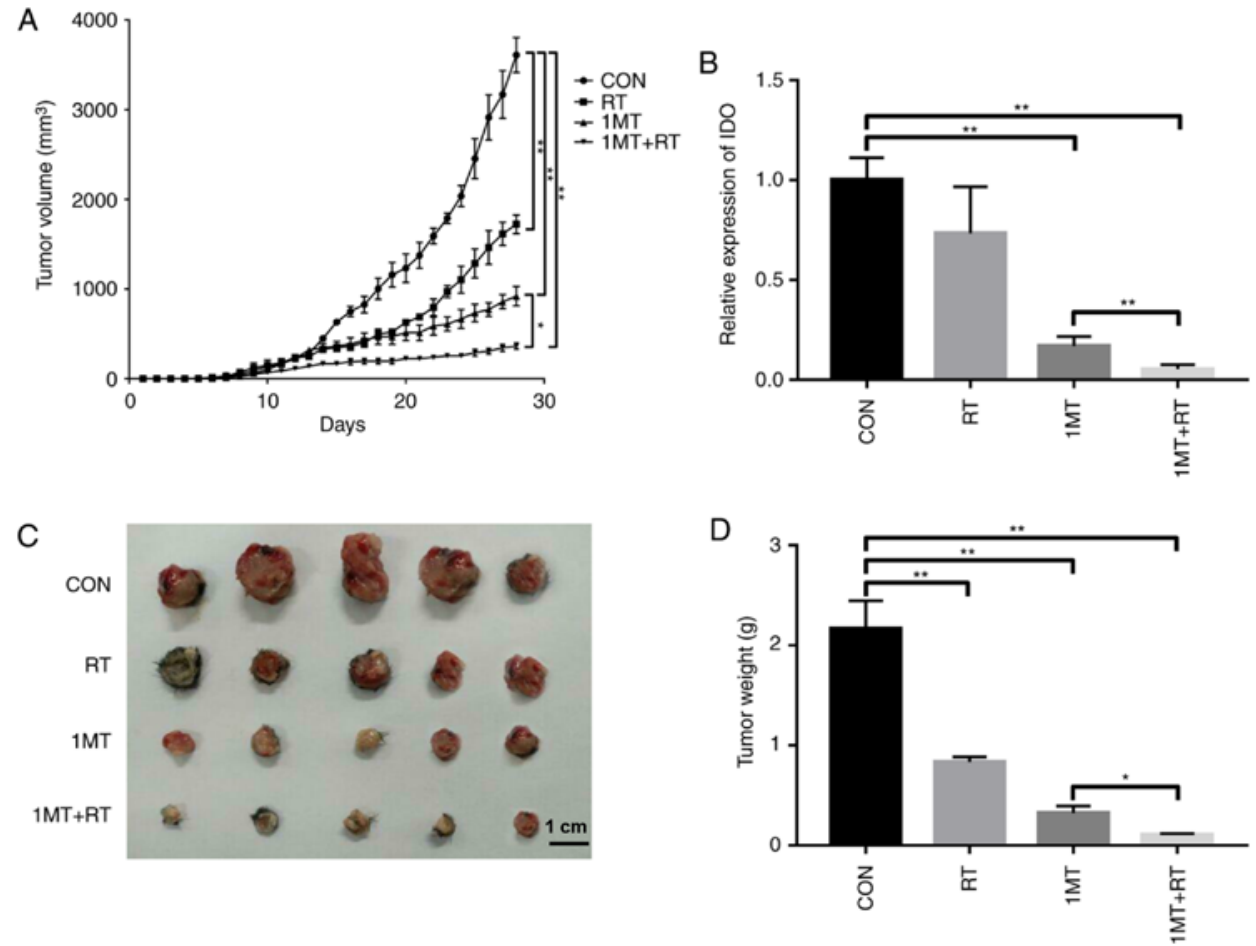

Figure 1. Tumor growth and IDO expression are reduced by combined treatment with 1MT and RT. (A) In vivo evaluation of IDO inhibition and RT in C57BL/6 female mice with tumors generated by the subcutaneous injection of LLC cells indicated that 1MT combined with RT significantly suppressed tumor growth and reduced tumor size compared with the single treatments. (B) Analysis of IDO expression in tumor cells revealed that IDO inhibition synergized with RT and significantly downregulated its expression. (C and D) Comparison of the average tumor weights indicated that tumor weight and volume were significantly reduced with RT and/or $1 \mathrm{MT}$, while the combined therapy showed the strongest effect. $\mathrm{n}=5$, experiments were repeated three times. ${ }^{* *} \mathrm{P}<0.001$, ${ }^{*} \mathrm{P}<0.01$ vs. CON. IDO, indoleamine 2,3-dioxygenase; RT, radiotherapy; 1MT, 1-methyl-tryptophan; CON, control.

DC maturation, enhancing their ability to activate T cells and to initiate the immune response. The IDO inhibitor synergized with RT to produce an increase in immune activation effects compared with the use of the treatments individually.
IDO inhibition synergizes with $R T$ to reduce the expression of inhibitory receptors and Tregs, and promote $T$ cell activation. Through stimulation by antigen peptide-MHC II complexes and co-stimulatory factors, DCs can activate $\mathrm{T}$ cells and 
A
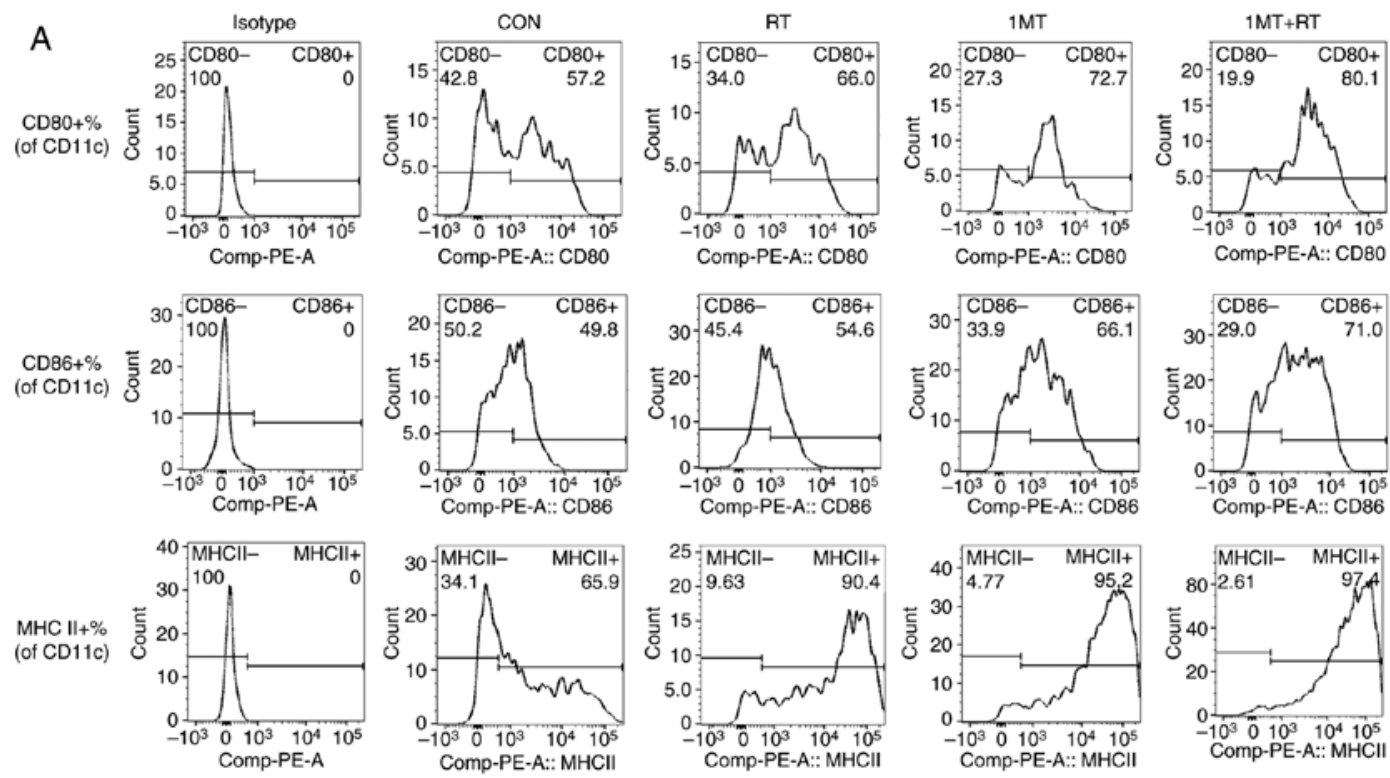
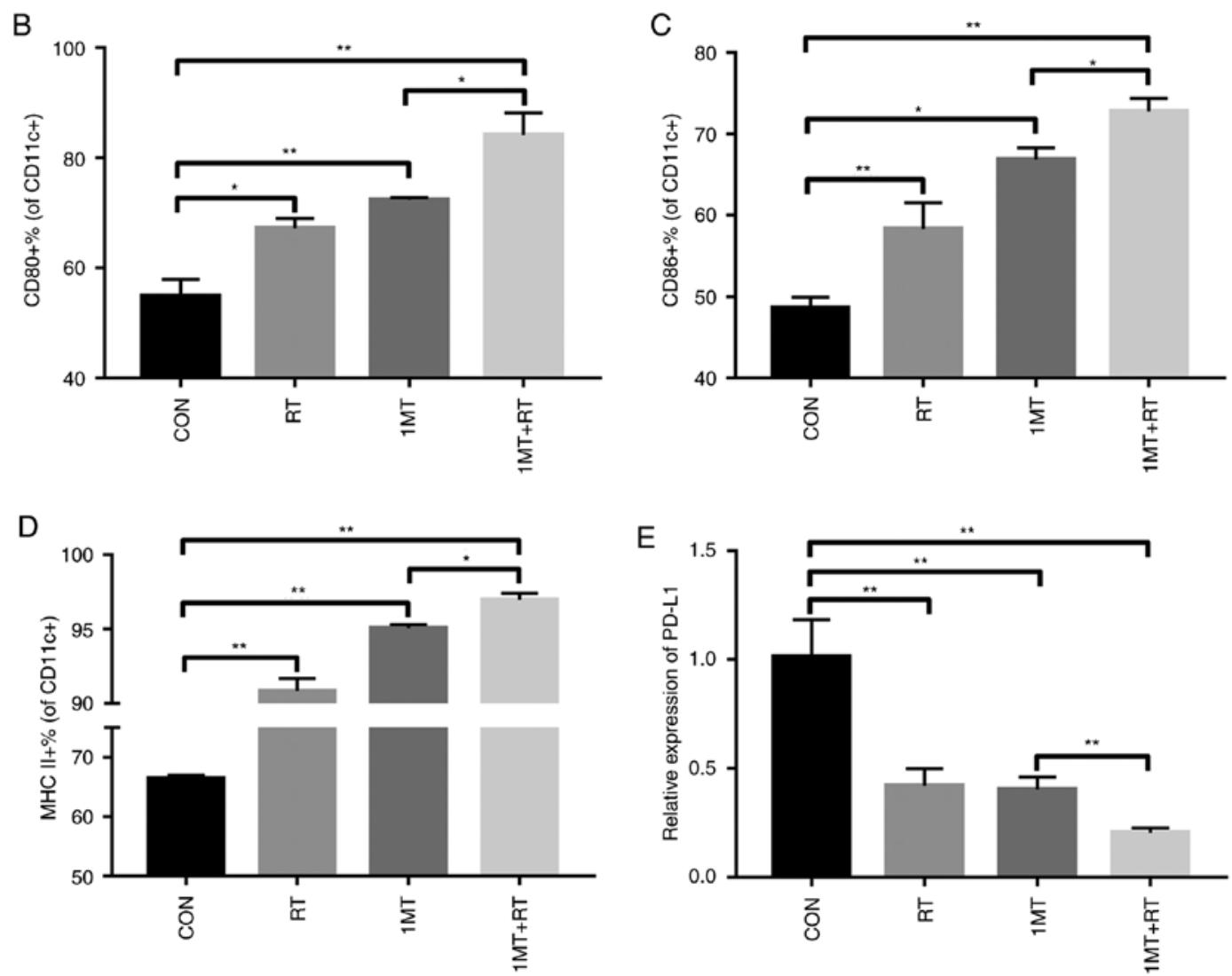

Figure 2. IDO inhibition and RT in combination promote the maturation of DCs and downregulate the inhibitory receptor ligand PD-L1 in the tumor microenvironment. (A) The results of flow cytometry. To validate the influences of changes in IDO expression on DCs, maturity markers, including CD80, CD86 and MHC II, were examined. Comparison of the maturation of DCs under various treatments based on flow cytometry. RT and IDO inhibitor treatment upregulated the expression of (B) CD80 and (C) CD86. The combined therapy of 1MT+RT showed the strongest effect, which significantly increased the expression of the cell surface co-stimulatory factors CD80 and CD86 compared with the single treatments. (D) Evaluation of the ability to initiate T cell activation and provide a second stimulatory signal was performed by measuring the expression level of MHC II on DCs. The results indicated that RT and IDO inhibitor treatment significantly upregulated the expression of MHC II compared with the CON group; the combined therapy showed the strongest effect. (E) PD-L1 in the tumor microenvironment, as an inhibitory receptor ligand expressed on DCs, was measured. The results suggested that RT and IDO inhibition significantly downregulated PD-L1 expression compared with the CON group; the $\mathrm{n}=5$, experiments were repeated three times. combined therapy had the strongest effect. ${ }^{* *} \mathrm{P}<0.001,{ }^{*} \mathrm{P}<0.01$ vs. CON. IDO, indoleamine 2,3-dioxygenase; RT, radiotherapy; 1MT, 1-methyl-tryptophan; CON, control; DCs, dendritic cells; MHC II, major histocompatibility complex II; PD-L1, programmed cell death ligand 1.

thus facilitate the clearance of antigens, and regulate the immune response (26). Therefore, through direct or indirect regulation of T cells, the IDO inhibitor can active the immune response against a tumor. As shown in the previous results, 
the IDO inhibitor can increase the maturity of DCs and downregulate the expression of inhibitory receptor ligands, which increases the ability of DCs to activate T cells. To determine whether this phenomenon can enhance the activity of $\mathrm{T}$ cells, IFN- $\gamma$, Tregs and inhibitory receptors were examined.

IFN- $\gamma$ is a dimeric soluble cy tokine with immuno-activating and antitumor properties (27). Based on the results of flow cytometry (Fig. 3A and B), compared with the CON group, IDO inhibition increased the activation and secretion of IFN- $\gamma$, suggesting that $1 \mathrm{MT}$ can enhance the differentiation of T cells, which can result in an enhanced antitumor ability. RT can also significantly upregulate the secretion of IFN- $\gamma$ compared with the CON group. The combined group, which was treated with both RT and the IDO inhibitor, showed a better therapeutic outcome than either individual treatment group.

Tregs, as a subpopulation of $\mathrm{T}$ cells, regulate the immune system by suppressing $\mathrm{T}$ cell activation and proliferation (28). Based on the results of flow cytometry analysis (Fig. 3A and C), compared with the CON group, IDO inhibition significantly decreased the number of Tregs, suggesting that $1 \mathrm{MT}$ can relieve the negative regulatory effect caused by Tregs and boost $\mathrm{T}$ cell activity. Thus, IDO inhibition can enhance $\mathrm{T}$ cell function and antitumor immunity. IDO inhibition synergized with RT to yield better therapeutic effects than either therapy alone.

PD-1 is a protein receptor that regulates the immune response by promoting self-tolerance and downregulating immunity (29). Based on the RT-qPCR analysis (Fig. 3D), no significant difference in PD-1 expression was found between the RT and CON groups, however, IDO inhibition significantly downregulated the expression of PD-1, and when IDO inhibition synergized with RT, the inhibitory effect was greater. This suggested that the IDO inhibitor synergized with RT, and that this relieves the inhibitory effects of PD-1 on T cells to a greater extent than either therapy alone. Therefore, combined therapy can activate $\mathrm{T}$ cells and boost antitumor immunity to a greater degree than either treatment alone.

TIM-3 is an immune checkpoint protein that promotes immune suppression together with other inhibitory molecules. TIM-3 is often upregulated in tumor-infiltrating lymphocytes, strategies targeting TIM-3 can be used to boost T cell activity and activate antitumor immunity (30). Based on the RT-qPCR analysis (Fig. 3E), compared with the CON group, RT alone had no effect on the expression of TIM-3, however, IDO inhibition significantly downregulated the expression of TIM-3, indicating that 1MT can relieve the inhibitory effects of TIM-3 on $\mathrm{T}$ cells and prevent $\mathrm{T}$ cell depletion. These results suggested that IDO inhibition boosted antitumor immunity. The IDO inhibitor synergized with RT to yield a better therapeutic effect than either therapy alone.

BTLA is a protein expressed on Thelper (Th) 1 cells during $\mathrm{T}$ cell activation and a negative regulatory ligand that inhibits T cells (31). Based on the RT-qPCR analysis (Fig. 3F), no significant difference in BTLA expression was found between the RT and CON groups, however, IDO inhibition decreased the expression level of BTLA compared with the CON group, which may result in enhanced $\mathrm{T}$ cell activity. Therefore, IDO inhibitors can enhance $\mathrm{T}$ cell activity and antitumor immunity. IDO inhibition synergized with RT and yielded better therapeutic effects than either treatment alone.
Galectin-9 is a ligand expressed on the surface of multiple types of cancer cells, and as the most studied ligand for TIM-3. Galectin-9 can lead to T cell exhaustion by inhibiting $\mathrm{T}$ cell proliferation and inducing apoptosis (32). Based on the RT-qPCR analysis (Fig. 3G), RT alone had no effect on the expression of galectin-9, however, IDO inhibition significantly downregulated the expression of galectin- 9 compared with the CON group, indicating that $1 \mathrm{MT}$ relieves the inhibitory effects of galectin-9 on T cells and boosts antitumor immunity. IDO inhibition synergized with RT and yielded better therapeutic effect than either treatment alone.

\section{Discussion}

The aim of the present study was to examine the feasibility and efficacy of RT combined with IDO inhibition in treating cancer.

The role of the inflammatory response in RT remains difficult to predict. The efficacy of RT is closely related to the immune status of the patient. RT can not only trigger innate and adaptive immune responses, leading to tumor regression, but can also cause tumor cells to develop a variety of resistance mechanisms that promote tumor immune escape (33-35), which is why RT can synergize with immunotherapy to produce greater effects on the inhibition of tumor growth, IDO and negative regulators. By analyzing the results of combination therapy, the positive effects of IDO inhibition can be observed. IDO is an intracellular enzyme that degrades the essential amino acid L-tryptophan to N-kynurenine (36). IDO exerts immunosuppressive effects by reducing the local concentration of tryptophan and increasing the production of immunomodulatory tryptophan metabolites (37). Immunomodulatory tryptophan metabolites have different effects on immune cells. For example, the metabolites can inhibit proliferation and promote the apoptosis of $\mathrm{T}$ lymphocytes, and can induce naive $\mathrm{T}$ cells to differentiate into Tregs. IDO overexpression in DCs can affect cell maturation, which results in decreased antigen presentation and the increased expression of negative co-stimulatory molecules (36). To alleviate the inhibitory effect of IDO on the immune system, the present study used an IDO inhibitor. Treatment with 1MT has been shown to enhance the function of cytotoxic lymphocytes in vitro following an allogeneic mixed lymphocyte reaction by reducing the expression of IDO (38). In addition, significant reductions in the levels of the associated negative regulators PD-1, TIM-3 and BTLA are also associated with the effects of RT and IDO inhibitors (39-41). PD-1 and its ligands, PD-L1 and PD-L2, are immunological checkpoint proteins whose primary function is to limit inflammatory reactions in peripheral tissues (42). However, when these proteins are expressed in the tumor microenvironment, this process represents an effective mechanism for tumor-induced immunosuppression and evasion. TIM-3 is a member of the TIM gene family, which includes TIM-1, TIM-3, and TIM-4 in humans and TIM-1-8 in mice. TIM-3 is expressed on Th1, Th17 and CD8 ${ }^{+}$ $\mathrm{T}$ cells of the mouse bone marrow lineage (43). Binding between TIM-3 and its ligand has been found to inhibit T cell responses and induce peripheral immune tolerance. BTLA is an inhibitory molecule expressed by T cells, B cells, DCs and natural killer cells. Herpes Virus Entering Medium is a 
A
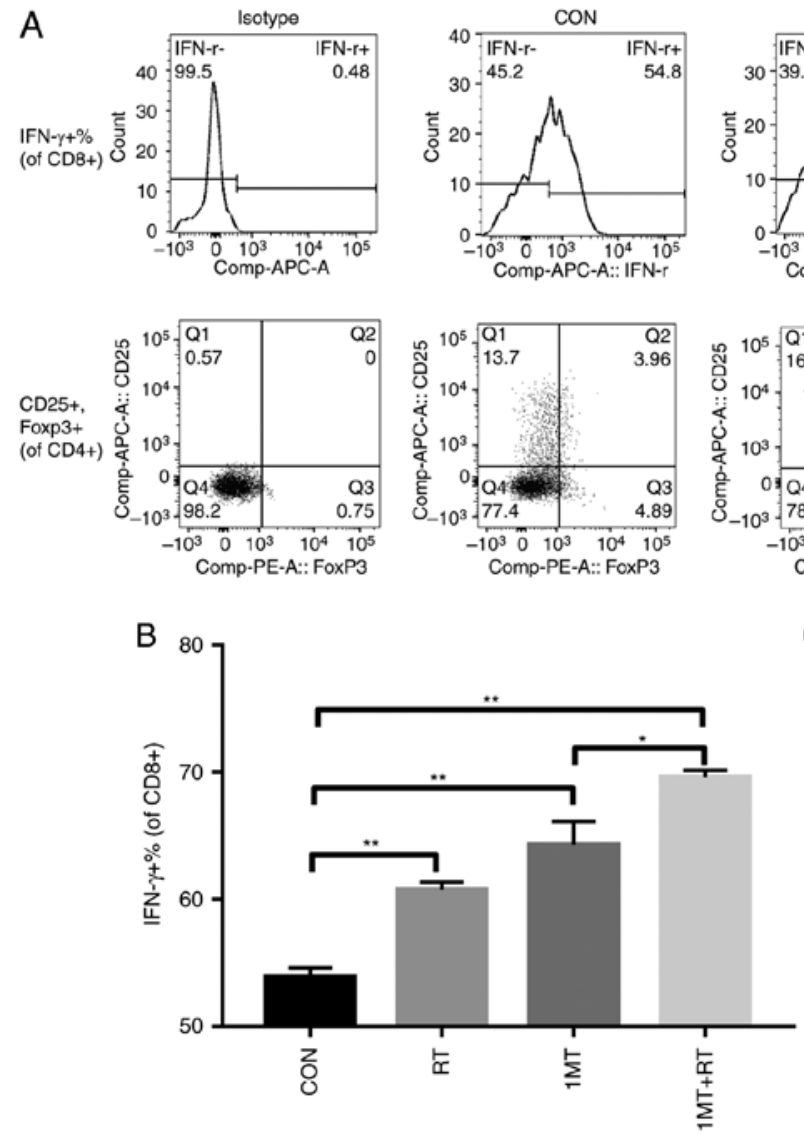

$\mathrm{D}$
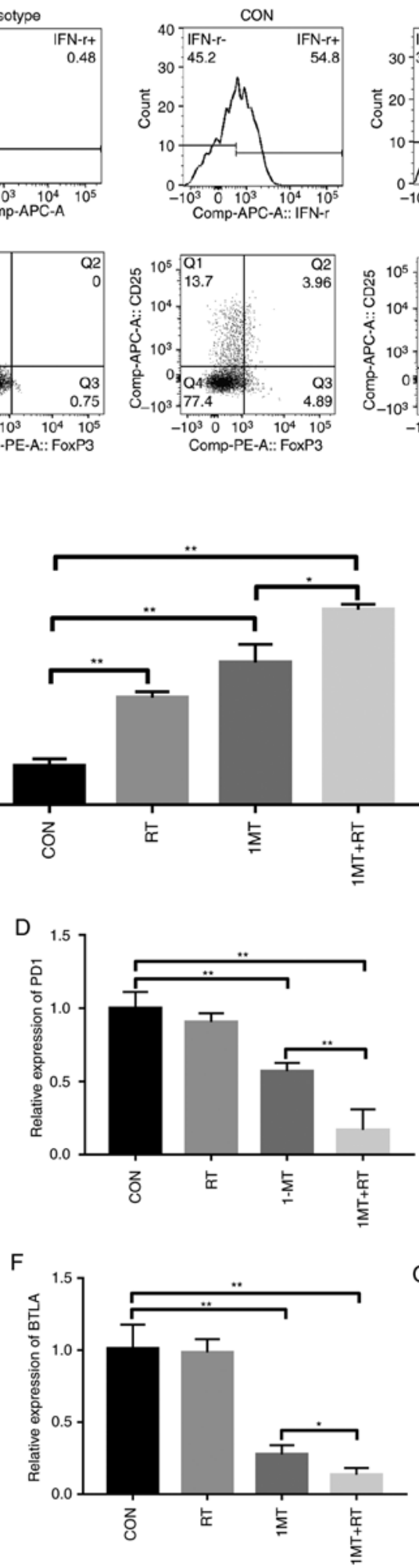
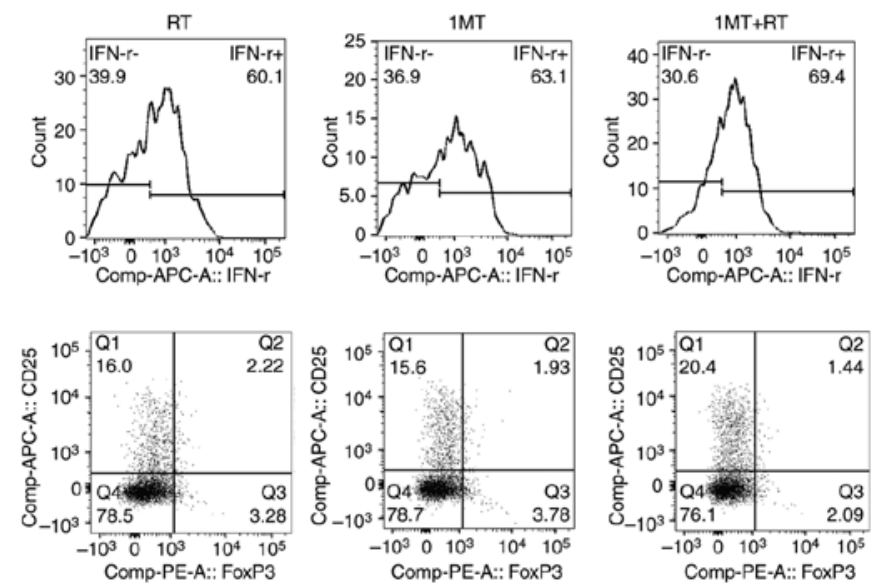

C
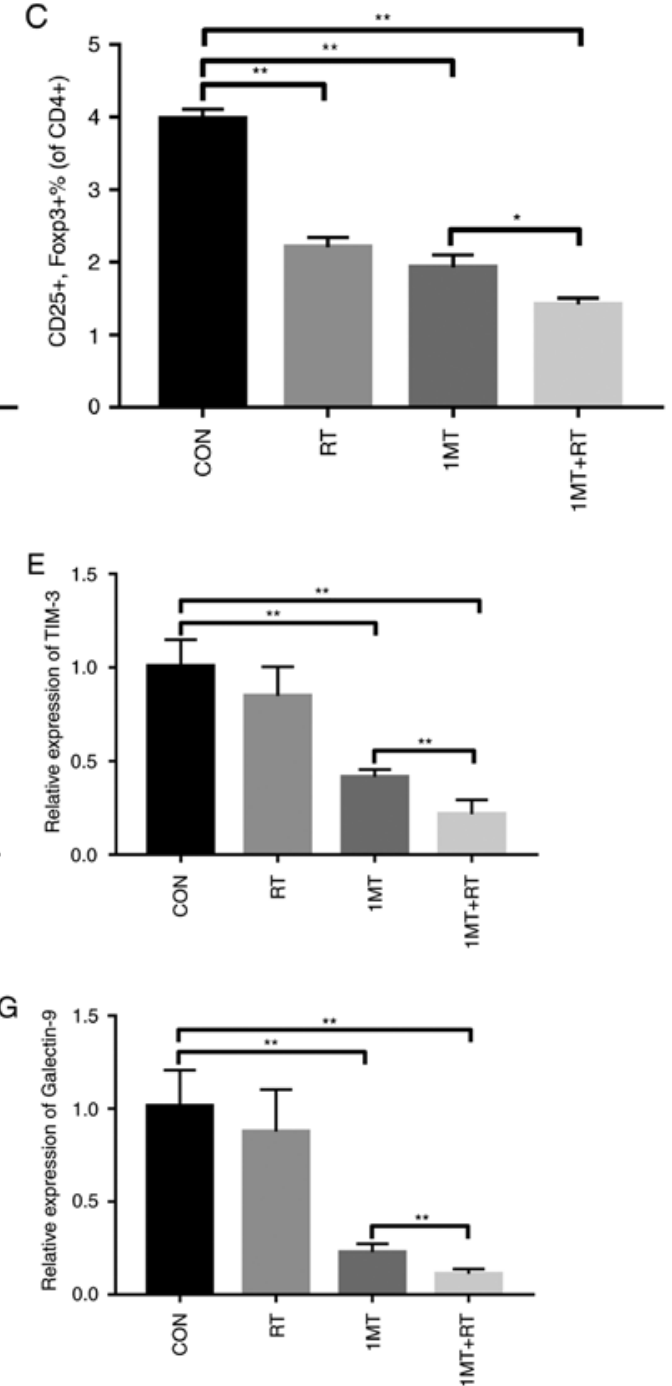

Figure 3. IDO inhibition and RT in combination reduce the expression of inhibitory receptors and Tregs, and promote T cell activation. (A) The results of flow cytometry. IFN- $\gamma$, a dimeric soluble cytokine that promotes the proliferation and activation of cytotoxic lymphocytes, was examined to determine whether the activity of $\mathrm{T}$ cells was enhanced following treatment. $\mathrm{CD} 4{ }^{+} \mathrm{CD} 25^{+} \mathrm{FOXP} 3^{+}$Tregs are also measured to determine the inhibitory effect on $\mathrm{T}$ cells. (B) The results showed that IDO inhibition synergized with RT to produce the most significant effect among all the treatments. The combined therapy of $1 \mathrm{MT}+\mathrm{RT}$ had the stronger therapeutic effect. (C) The percentage of $\mathrm{CD} 4^{+} \mathrm{CD} 25^{+} \mathrm{FOXP} 3^{+}$cells $\left(\mathrm{CD} 25^{+} \mathrm{FOXP} 3^{+}\right.$cells of $\mathrm{CD} 4^{+}$cells) was measured to evaluate the inhibitory effect on Tregs. The results indicated that combined therapy showed the strongest inhibitory effect on Tregs. PD-1, TIM-3 and BTLA are negative regulators expressed on T cells and together lead to T cell anergy and exhaustion. The levels of (D) PD-1, (E) TIM-3 and (F) BTLA were measured, the results indicated that there was no significant difference between the CON and RT groups, however, 1MT and 1MT combined with RT significantly decreased the expression of these markers compared with the CON group; the combined group showing the strongest effect. (G) Galectin-9 is expressed on cancer cells and is responsible for inhibition of $\mathrm{T}$ cell proliferation, and apoptosis. The level of galectin- 9 was measured and the results showed no significant difference between the CON and RT groups, however, 1MT significantly downregulated galectin-9 expression compared with the CON group; IDO inhibition synergized with RT to produce the stronger effect on Galecton-9 expression. $\mathrm{n}=5$, experiments were repeated three times. ${ }^{* *} \mathrm{P}<0.001$, ${ }^{*} \mathrm{P}<0.01$ vs. CON group. IDO, indoleamine 2,3 -dioxygenase; RT, radiotherapy; 1MT, 1-methyl-tryptophan; CON, control; PD-1, programmed cell death 1; TIM-3, T cell immunoglobulin domain and mucin domain 3; BTLA, B- and T-lymphocyte attenuator; IFN- $\gamma$, interferon- $\gamma$; FOXP3, Forkhead box P3; Tregs, regulatory T cells. 
known ligand for BTLA. The cytoplasmic domain of BTLA is required for BTLA to fully inhibit $\mathrm{T}$ cell proliferation and cytokine production, including IFN- $\gamma$, IL-2 and IL-10. The reduction of these cytokines may be related to the reduction of IDO. These molecules have similar functions in promoting $\mathrm{T}$ cell activation and DC-mediated activation of T cells, and enhancing immunity (44), which is consistent with the results of the tumor growth curve in the present study showing that tumor growth was significantly reduced.

The present study found that $1 \mathrm{MT}$ combined with RT caused greater reductions in tumor growth, decreases in inhibitory receptors and ligands, and activation of DCs and T cells in vivo compared with either therapy alone. The killing activity of the activated cytotoxic $\mathrm{T}$ lymphocytes may further indicate the immune activation ability of this therapeutic strategy. In future experiments, additional measurements will be made during in vitro experiments, including measurement of the killing activity of cytotoxic T lymphocytes.

Jiang and Chan (45) summarized the effects of RT and immune checkpoint blockade, supporting the results of the present study. Other in vitro experiments with tumor cells treated with RT or IDO inhibitors also showed similar effects, which also support the findings of the present study (46).

In conclusion, the present study demonstrated that IDO inhibition combined with RT can significantly decrease the expression level of IDO in tumor cells compared with the CON group. Furthermore, DCs tend to be mature and show a greater antigen-presenting ability following combination therapy. Inhibitory receptors and ligands were downregulated in both DCs and T cells following combined therapy compared with the CON group, which partially overcomes the problem of $\mathrm{T}$ cell exhaustion. Together, these changes facilitate the activation of antitumor immunity. In addition, IDO inhibition and RT synergized to provide a more effective inhibition of tumor growth than either therapy alone.

\section{Acknowledgements}

Not applicable.

\section{Funding}

The present study was supported by grants from National Natural Science Foundation of China (grant no. 81860504) and the Key Research and Development Program of Jiangxi Province (grant no. 20171BBG70120).

\section{Availability of data and materials}

All data generated or analyzed during the present study are included in this published article.

\section{Authors' contributions}

HW, ML, ZL, WY, XZ, LW, JC, BM, RZ and WM were involved in the conception and supervision of the project. $\mathrm{ML}$ and $\mathrm{ZL}$ were involved in the design of the study. ML and ZL performed the experiments, analyzed the results, and prepared the paper. All the authors read and approved the final manuscript.

\section{Ethics approval and consent to participate}

All animal experiments were performed in accordance with the Animal Research Reporting of in vivo Experiments and the National Institutes of Health guidelines for animal welfare, and the study was approved by the Institutional Animal Care and Use Committee of Nanchang University, China. All animal experiments complied with the ARRIVE guidelines and the AVMA euthanasia guidelines of 2013.

\section{Patient consent for publication}

Not applicable.

\section{Competing interests}

The authors declare that they have no competing interests.

\section{References}

1. O'Donnell JS, Smyth MJ and Teng MW: Acquired resistance to anti-PD1 therapy: Checkmate to checkpoint blockade? Genome Med 8: 111, 2016.

2. Weinlich R, Oberst A, Beere HM and Green DR: Necroptosis in development, inflammation and disease. Nat Rev Mol Cell Biol 18: 127-136, 2017.

3. Postow MA, Callahan MK, Barker CA, Yamada Y, Yuan J, Kitano S, Mu Z, Rasalan T, Adamow M, Ritter E, et al: Immunologic correlates of the abscopal effect in a patient with melanoma. N Engl J Med 366: 925-931, 2012.

4. Levy A, Chargari C, Marabelle A, Perfettini JL, Magné N and Deutsch E: Can immunostimulatory agents enhance the abscopal effect of radiotherapy? Eur J Cancer 62: 36-45, 2016.

5. Golden EB, Chhabra A, Chachoua A, Adams S, Donach M, Fenton-Kerimian M, Friedman K, Ponzo F, Babb JS, Goldberg J, et al: Local radiotherapy and granulocyte-macrophage colony-stimulating factor to generate abscopal responses in patients with metastatic solid tumours: A proof-of-principle trial. Lancet Oncol 16: 795-803, 2015.

6. Seyedin SN, Tang C and Welsh JW: Author's view: Radiation and immunotherapy as systemic therapy for solid tumors. Oncoimmunology 4: e986402, 2015.

7. Ungaro A, Orsi F, Casadio C, Galdy S, Spada F, Cella CA, Tonno CD, Bonomo G, Vigna PD, Murgioni S, et al: Successful palliative approach with high-intensity focused ultrasound in a patient with metastatic anaplastic pancreatic carcinoma: A case report. Ecancermedicalscience 10: 635, 2016.

8. Espenel S, Vallard A, Rancoule C, Garcia MA, Guy JB, Chargari C, Deutsch E and Magné N: Melanoma: Last call for radiotherapy. Crit Rev Oncol Hematol 110: 13-19, 2017.

9. Lu CS and Liu JH: Pneumonitis in cancer patients receiving anti-PD-1 and radiotherapies: Three case reports. Medicine (Baltimore) 96: e5747, 2017.

10. Ye Q, Wang C, Xian J, Zhang M, Cao Y and Cao Y: Expression of programmed cell death protein 1 (PD-1) and indoleamine 2,3-dioxygenase (IDO) in the tumor microenvironment and in tumor-draining lymph nodes of breast cancer. Hum Pathol 75: 81-90, 2018.

11. Liang C, Peng L, Zeng S, Zhao Q, Tang L, Jiang X, Zhang J, Yan $\mathrm{N}$ and Chen Y: Investigation of indoleamine 2,3-dioxygenase 1 expression in uveal melanoma. Exp Eye Res 181: 112-119, 2019.

12. Brahmer J, Reckamp KL, Baas P, Crinó L, Eberhardt WE, Poddubskaya E, Antonia S, Pluzanski A, Vokes EE, Holgado E, et al: Nivolumab versus docetaxel in advanced squamous-cell non-small-cell lung cancer. N Engl J Med 373: 123-135, 2015.

13. Younes A, Brody J, Carpio C, Lopez-Guillermo A, Ben-Yehuda D, Ferhanoglu B, Nagler A, Ozcan M, Avivi I, Bosch F, et al: Safety and activity of ibrutinib in combination with nivolumab in patients with relapsed non-Hodgkin lymphoma or chronic lymphocytic leukaemia: A phase 1/2a study. Lancet Haematol 6: e67-e78, 2019. 
14. Prendergast GC, Malachowski WJ, Mondal A, Scherle P and Muller AJ: Indoleamine 2,3-dioxygenase and its therapeutic inhibition in cancer. Int Rev Cell Mol Biol 336: 175-203, 2018.

15. Kozuma Y, Takada K, Toyokawa G, Kohashi K, Shimokawa M, Hirai F, Tagawa T, Okamoto T, Oda Y and Maehara Y: Indoleamine 2,3-dioxygenase 1 and programmed cell death-ligand 1 co-expression correlates with aggressive features in lung adenocarcinoma. Eur J Cancer 101: 20-29, 2018.

16. Li A, Barsoumian HB, Schoenhals JE, Cushman TR, Caetano MS, Wang X, Valdecanas DR, Niknam S, Younes AI, $\mathrm{Li} \mathrm{G}$, et al: Indoleamine 2,3-dioxygenase 1 inhibition targets anti-PD1-resistant lung tumors by blocking myeloid-derived suppressor cells. Cancer Lett 431: 54-63, 2018

17. Tanizaki Y, Kobayashi A, Toujima S, Shiro M, Mizoguchi M, Mabuchi Y, Yagi S, Minami S, Takikawa $\mathrm{O}$ and Ino $\mathrm{K}$ : Indoleamine 2,3-dioxygenase promotes peritoneal metastasis of ovarian cancer by inducing an immunosuppressive environment. Cancer Sci 105: 966-973, 2014.

18. Moreno ACR, Porchia B, Pagni RL, Souza PDC, Pegoraro R, Rodrigues KB, Barros TB, Aps LRMM, de Araújo EF, Calich VLG and Ferreira LCS: The combined use of melatonin and an indoleamine 2,3-dioxygenase-1 inhibitor enhances vaccine-induced protective cellular immunity to HPV16-associated tumors. Front Immunol 9: 1914, 2018.

19. Alahdal M, Xing Y, Tang T and Liang J: 1-Methyl-D-tryptophan reduces tumor $\mathrm{CD} 133^{+}$cells, Wnt/ $\beta$-catenin and NF-kappaßp65 while enhances lymphocytes NF- $\kappa \beta 2$, STAT3, and STAT4 pathways in murine pancreatic adenocarcinoma. Sci Rep 8: 9869, 2018.

20. Spranger S, Spaapen RM, Zha Y, Williams J, Meng Y, Ha TT and Gajewski TF: Up-regulation of PD-L1, IDO, and T(regs) in the melanoma tumor microenvironment is driven by CD8(+) T cells. Sci Transl Med 5: 200ra116, 2013.

21. Williams DK, Markwalder JA, Balog AJ, Chen B, Chen L, Donnell J, Haque L, Hart AC, Mandal SK, Nation A, et al: Development of a series of novel o-phenylenediamine-based indoleamine 2,3-dioxygenase 1 (IDO1) inhibitors. Bioorg Med Chem Lett 28: 732-736, 2018

22. Vatner RE, Cooper BT, Vanpouille-Box C, Demaria S and Formenti SC: Combinations of immunotherapy and radiation in cancer therapy. Front Oncol 4: 325, 2014.

23. Jia H, Ren W, Feng Y, Wei T, Guo M, Guo J, Zhao J, Song X, Wang M, Zhao T, et al: The enhanced antitumour response of pimozide combined with the IDO inhibitor L-MT in melanoma. Int J Oncol 53: 949-960, 2018.

24. Leary S, Underwood W, Anthony R, Cartner S, Corey D, Grandin T, Greenacre C, Gwaltney-Brant S, McCrackin MA, Meyer R, et al: AVMA Guidelines for the Euthanasia of Animals: 2013 edition. University of Alaska Anchorage, 2013, 2013.

25. Livak KJ and Schmittgen TD: Analysis of relative gene expression data using real-time quantitative PCR and the 2(-Delta Delta C(T)) method. Methods 25: 402-408, 2001.

26. Holling TM, Schooten E and van Den Elsen PJ: Function and regulation of MHC class II molecules in T-lymphocytes: Of mice and men. Hum Immunol 65: 282-290, 2004.

27. Kursunel MA and Esendagli G: The untold story of IFN- $\gamma$ in cancer biology. Cytokine Growth Factor Rev 31: 73-81, 2016.

28. Gondek DC, Lu LF, Quezada SA, Sakaguchi S and Noelle RJ: Cutting edge: Contact-mediated suppression by CD4+CD25+ regulatory cells involves a granzyme B-dependent, perforin-independent mechanism. J Immunol 174: 1783-1786, 2005.

29. Fife BT and Pauken KE: The role of the PD-1 pathway in autoimmunity and peripheral tolerance. Ann N Y Acad Sci 1217: 45-59, 2011.

30. Blackburn SD, Shin H, Haining WN, Zou T, Workman CJ, Polley A Betts MR, Freeman GJ, Vignali DA and Wherry EJ: Coregulation of CD8+ T cell exhaustion by multiple inhibitory receptors during chronic viral infection. Nat Immunol 10: 29-37, 2009.
31. Haymaker CL, Wu RC, Ritthipichai K, Bernatchez C, Forget MA, Chen JQ, Liu H, Wang E, Marincola F, Hwu P and Radvanyi LG: BTLA marks a less-differentiated tumor-infiltrating lymphocyte subset in melanoma with enhanced survival properties. Oncoimmunology 4: e1014246, 2015.

32. Sakuishi K, Apetoh L, Sullivan JM, Blazar BR, Kuchroo VK and Anderson AC: Targeting Tim-3 and PD-1 pathways to reverse $\mathrm{T}$ cell exhaustion and restore anti-tumor immunity. J Exp Med 207: 2187-2194, 2010.

33. Vanpouille-Box C, Alard A, Aryankalayil MJ, Sarfraz Y, Diamond JM, Schneider RJ, Inghirami G, Coleman CN, Formenti SC and Demaria S: DNA exonuclease Trex1 regulates radiotherapy-induced tumour immunogenicity. Nat Commun 8: $15618,2017$.

34. Gong X, Li X, Jiang T, Xie H, Zhu Z, Zhou F and Zhou C: Combined radiotherapy and Anti-PD-L1 antibody synergistically enhances antitumor effect in non-small cell lung cancer. J Thorac Oncol 12: 1085-1097, 2017.

35. Herter-Sprie GS, Koyama S, Korideck H, Hai J, Deng J, Li YY, Buczkowski KA, Grant AK, Ullas S, Rhee K, et al: Synergy of radiotherapy and PD-1 blockade in Kras-mutant lung cancer. JCI Insight 1: e87415, 2016.

36. Brown ZJ, Yu SJ, Heinrich B, Ma C, Fu Q, Sandhu M, Agdashian D, Zhang Q, Korangy $\mathrm{F}$ and Greten TF: Indoleamine 2,3-dioxygenase provides adaptive resistance to immune checkpoint inhibitors in hepatocellular carcinoma. Cancer Immunol Immunother 67: 1305-1315, 2018.

37. Lim JY, Lee SE, Park G, Choi EY and Min CK: Inhibition of indoleamine 2,3-dioxygenase by stereoisomers of 1-methyl tryptophan in an experimental graft-versus-tumor model. Exp Hematol 42: 862-866.e3, 2014.

38. Xiao B, Liu B, Song Y, Yu Z and Guo S: Local cytotoxic T-lymphocyte-associated antigen-4 immunoglobulin inhibition of rejection response is dependent on indoleamine 2,3-dioxygenase activities in the allograft. Transplant Proc 46: 3637-3640, 2014.

39. Ostrand-Rosenberg S, Horn LA and Ciavattone NG: Radiotherapy both promotes and inhibits myeloid-derived suppressor cell function: Novel strategies for preventing the tumor-protective effects of radiotherapy. Front Oncol 9: 215, 2019.

40. Oweida A, Hararah MK, Phan A, Binder D, Bhatia S, Lennon S, Bukkapatnam S, Van Court B, Uyanga N, Darragh L, et al: Resistance to radiotherapy and PD-L1 blockade is mediated by TIM-3 upregulation and regulatory T-cell infiltration. Clin Cancer Res 24: 5368-5380, 2018.

41. Xu JF, Zhao K, Huang BJ, Xiong P, Fang M, Shen GX and Gong FL: Clone and expression of murine BTLA extracellular domain gene and its effect on the expression of B7 on dendritic cells. Xi Bao Yu Fen Zi Mian Yi Xue Za Zhi 22: 413-416, 2006 (In Chinese)

42. Shukuya T and Carbone DP: Predictive markers for the efficacy of Anti-PD-1/PD-L1 antibodies in lung cancer. J Thorac Oncol 11: 976-988, 2016.

43. Du W, Yang M, Turner A, Xu C, Ferris RL, Huang J, Kane LP and $\mathrm{Lu} \mathrm{B}$ : TIM-3 as a target for cancer immunotherapy and mechanisms of action. Int J Mol Sci 18: pii: E645, 2017.

44. Ritthipichai K, Haymaker CL, Martinez M, Aschenbrenner A, Yi X, Zhang M, Kale C, Vence LM, Roszik J, Hailemichael Y, et al: Multifaceted role of BTLA in the control of CD8 ${ }^{+}$T-cell Fate after antigen encounter. Clin Cancer Res 23: 6151-6164, 2017.

45. Jiang W, Chan CK, Weissman IL, Kim BYS and Hahn SM: Immune priming of the tumor microenvironment by radiation. Trends Cancer 2: 638-645, 2016.

46. Hirata E and Sahai E: Tumor microenvironment and differential responses to therapy. Cold Spring Harb Perspect Med 7: pii: a026781, 2017. 